\section{LA-UR- 02-2012}

Approved for public release; distribution is unlimited.
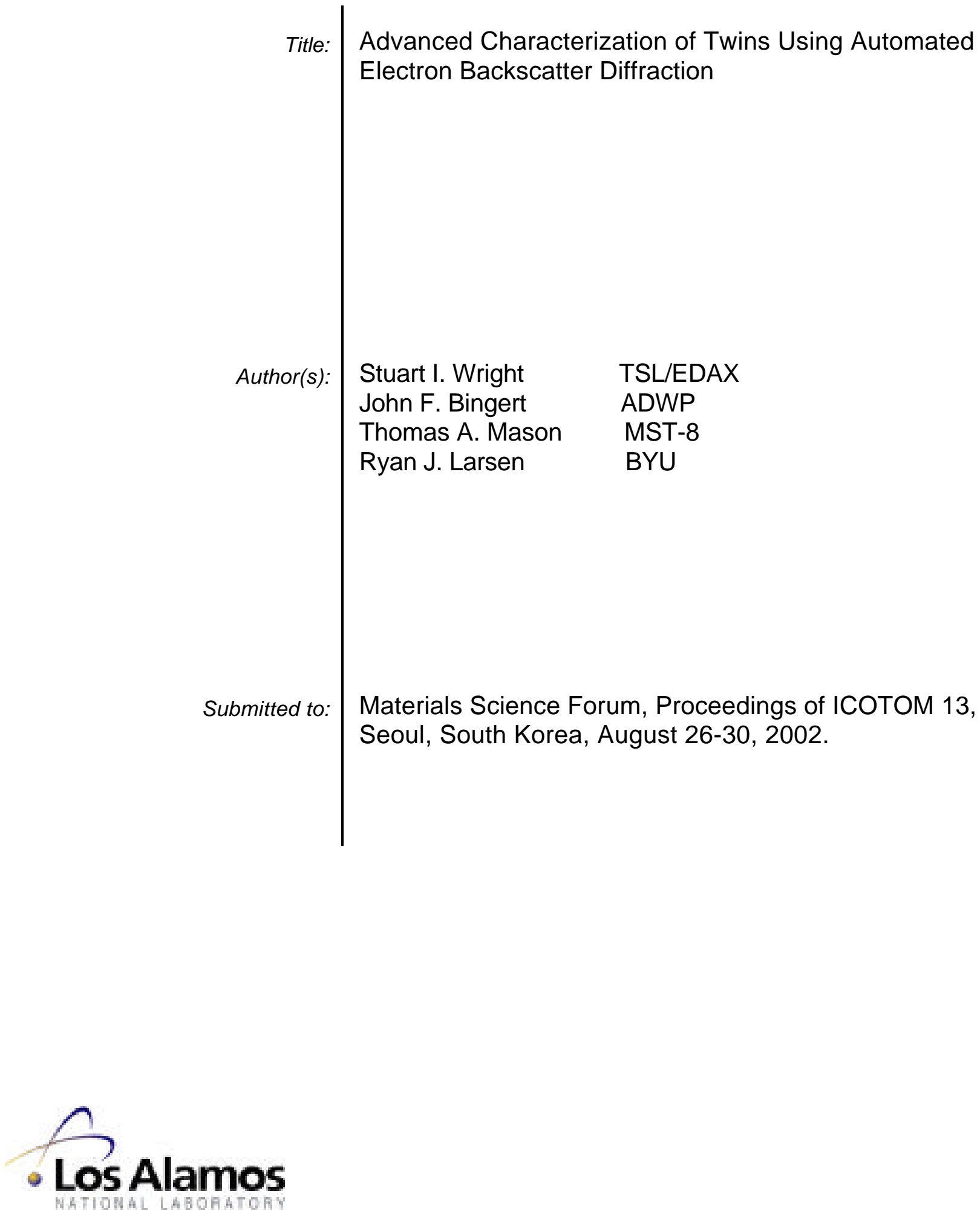

Los Alamos National Laboratory, an affirmative action/equal opportunity employer, is operated by the University of California for the U.S. Department of Energy under contract W-7405-ENG-36. By acceptance of this article, the publisher recognizes that the U.S. Government retains a nonexclusive, royaltyfree license to publish or reproduce the published form of this contribution, or to allow others to do so, for U.S. Government purposes. Los Alamos National Laboratory requests that the publisher identify this article as work performed under the auspices of the U.S. Department of Energy. Los Alamos National Laboratory strongly supports academic freedom and a researcher's right to publish; as an institution, however, the Laboratory does not endorse the viewpoint of a publication or guarantee its technical correctness. 


\title{
Advanced Characterization of Twins using Automated Electron Backscatter Diffraction
}

\author{
Stuart I. Wright ${ }^{1}$, John Bingert ${ }^{2}$, Thomas A. Mason ${ }^{3}$ and Ryan J. Larson ${ }^{4}$ \\ ${ }^{1}$ EDX/TSL, 392 E 12300 S, Draper, UT 84020 USA \\ ${ }^{2}$ Naval Research Lab, Code 6352, Multifunctional Materials Branch, BIdg. 28. Rm. 205, 4555 \\ Overlook Ave. SW, Washington DC 20375-5343 USA \\ ${ }^{3}$ Los Alamos National Laboratory, MST Division, Los Alamos, NM 87544 USA \\ ${ }^{4}$ Brigham Young University, Dept. of Mechanical Engineering, 435 CTB, Provo, UT 84602 USA
}

Keywords: Deformation Twinning, Electron Backscatter Diffraction (EBSD), Orientation Imaging Microscopy (OIM).

\begin{abstract}
This paper describes results obtained using an automated, crystallographically-based technique for twin identification. The technique is based on the automated collection of spatially specific orientation measurements by electron backscatter diffraction (EBSD) in the scanning electron microscope (SEM). The key features of the analysis are identification of potential twin boundaries by their misorientation character, identification of the distinct boundary planes among the symmetrically equivalent candidates, and validation of these boundaries through comparison with the boundary and twin plane traces in the sample cross section. Results on the application of this technique to deformation twins in zirconium are analyzed for the effect of twin type and amount and sense of uniaxial deformation. The accumulation of strain tends to increase the misorientation deviation at least to the degree of the trace deviation compared with recrystallization twins in nickel. In addition to the results on characterizing the twin character, results on extending the twin analysis to automated identification of parent and daughter material for structures exhibiting twin deformation are reported as well.
\end{abstract}

\section{Introduction}

The mapping of crystallographic orientations in polycrystals using automated electron backscatter diffraction (EBSD) systems provides a means for studying grain boundary character. This technique, sometimes termed Orientation Imaging Microscopy (OIM) [1], has been used to perform statistical characterizations of grain boundaries (for example see [2-5]). Most of these studies have focused on misorientation relationships. However, the misorientation provides only a partial description of a boundary. Another descriptive parameter is the orientation of the boundary plane both with respect to the sample reference frame as well as with respect to the crystal lattice of the adjoining grains. Randle $[3,6]$ has reviewed the need to consider both the misorientation at the boundary as well as the orientation of the boundary plane itself relative to the adjoining crystal lattices.

When considering twin boundaries, a key aspect of the boundary plane orientation is the relationship between the orientation of the boundary plane and the twinning plane. For example consider the primary recrystallization twin in face centered cubic materials. This boundary corresponds to a $60^{\circ}$ rotation about the $\langle 111\rangle$ crystal direction. For such a boundary to be considered a coherent twin not only must the misorientation between the grain separated by the 
boundary be near the ideal misorientation of $60^{\circ}$ about $\langle 111\rangle$; but, the boundary plane must also be oriented so that it is aligned with the $\{111\}$ planes in the adjoining crystals.

While OIM makes it relatively easy to measure grain boundary misorientations, the measurement of boundary plane orientations is not as straightforward. The orientation of individual grain boundary planes can be measured using either serial sectioning [5] or by observation of grain boundaries appearing on two mutually perpendicular sections [7] (i.e. at the sample edges). A statistical distribution of boundary plane orientations can also be obtained using stereology by sectioning on oblique planes[8]. While such studies can provide a complete description of all five geometrical boundary parameters (three for the misorientation and two the boundary plane orientation), they are difficult to perform. However, Wright and co-workers $[9,10]$ have shown that a partial description of boundary plane orientations can be obtained from OIM measurements on a single surface plane. This technique extends that described by Randle [11] by matching the poles of the misorientation-coincident planes.

Two critical parameters will be used in this study to characterize twin boundaries. The first is the misorientation deviation or $\Delta \theta$, which describes the angular deviation between the measured misorientation and the ideal twin misorientation. The second parameter is the trace deviation, $\Delta \omega$. The trace deviation is the angular distance between the trace of the boundary on the measured surface and the intersection the crystallographic twinning plane makes with the sample surface. An example of the trace deviation measure for two boundaries meeting the misorientation relationship criterion for a twin boundary is shown in Fig. 1. The sample is nickel and delineated boundaries correspond to the primary recrystallization twin $\left(60^{\circ}\right.$ about $\left.<111>\right)$. In this figure, the white boundaries are those which have both $\Delta \theta$ and $\Delta \omega$ values less than $8.7^{\circ}$ (the $\Delta \theta$ value prescribed by Brandon [12] for this type of boundary). The black boundaries are those boundaries with $\Delta \theta$ less than $8.7^{\circ}$ but with $\Delta \omega$ values greater than $8.7^{\circ}$ degrees. Two specific boundaries have been highlighted in the figure. The boundary marked A satisfies both criteria $-\Delta \omega$ is $0.6^{\circ}$. Boundary $\mathrm{B}$ does not meet the trace deviation criterion - $\Delta \omega$ equals $13^{\circ}$. The spoked figures overlaid on the map show the traces of $\{111\}$ boundaries within the grain. The "spokes" highlighted in white correspond to the pair of $\{111\}$ planes whose poles are most closely oriented, that is, those whose normals represent the common misorientation axis. If the proposition to be checked is whether or not the trace represents the actual coherent twin, then it is these specific planes from the family of crystallographic equivalents that should be considered for deviation from the trace. The approach for deformation twin boundary identification and verification is similar, but in this case the potential twinning plane is fixed for each system, and the proposition is whether or not the candidate boundary is actually a twin. 


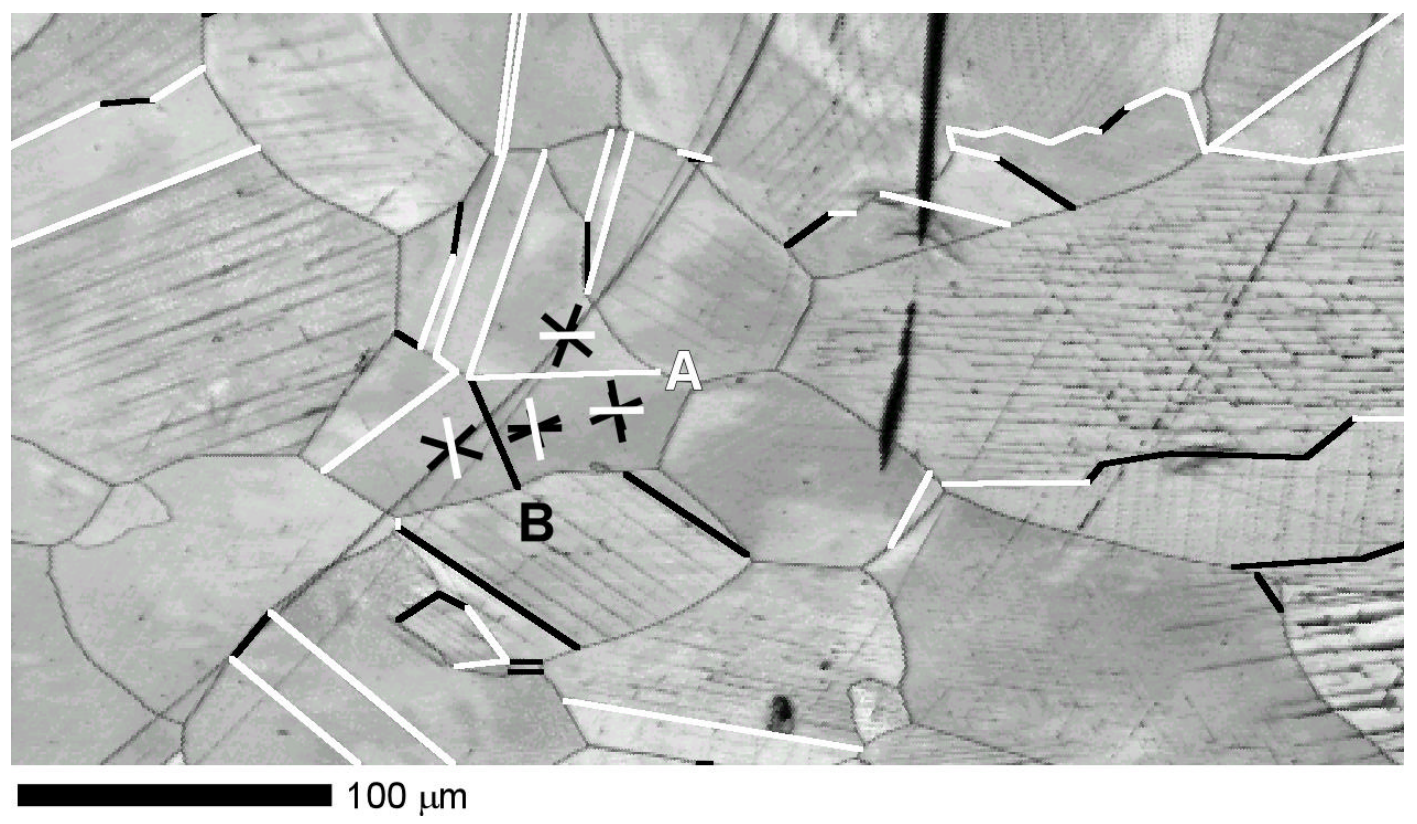

Figure 1 - Image quality map from nickel. The black and white highlighted boundaries satisfy the twin misorientation relationship. The white boundaries have $\{111\}$ twinning plane traces closely aligned with the boundary trace. The "spoked" figures show the $\{111\}$ plane traces.

This paper reports on $\Delta \theta, \Delta \omega$ results obtained using this advanced twin characterization technique. The effect of strain and twin type on deformation twin behavior will be examined, along with a comparison to results from a material exhibiting recrystallization twins.

\section{Deformation Twins in Zirconium}

OIM scans were performed on three zirconium samples: 1) 4\% true strain in compression 2) 10 $\%$ true strain in compression and 3) $16 \%$ true strain in tension. All three samples are from a bend beam tested at $77 \mathrm{~K}$ with the initial textures being equivalent [13-15]. OIM scans were performed on all three samples with step sizes of 1) $0.5 \mu \mathrm{m}$, 2) $0.7 \mu \mathrm{m}$ and 3) $0.4 \mu \mathrm{m}$. The number of points measured in each scan was 1) $138,000,2) 94,000$ and 3) 108,000. Image quality maps for the three samples are shown in Fig. 2.

Three types of deformation twins were characterized. A) The $\{10 \overline{1} 2\}$ tensile twin, identified by a rotation of $94.8^{\circ}$ about $<\overline{1} 2 \overline{1} 0>$; B) the $\{11 \overline{2} 1\}$ tensile twin of $34.8^{\circ}$ about $\langle\overline{1} 100>$; and $\mathrm{C}$ ) the $\{11 \overline{2} 2\}$ compressive twin of $64.3^{\circ}$ about $<\overline{1} 100>$. The twins are identified by their twinning plane. The twinning plane is very nearly identical to the composition plane whose trace is actually observed, at least at small strains. Table 1 shows the candidate fraction of these types of twins found in each sample based solely on the misorientation relationship. Since for these deformation twins the misorientation axis is not normal to the twin plane, a check on coincidence of the twin plane normals will eliminate some of the spurious boundaries at this stage [15]. 


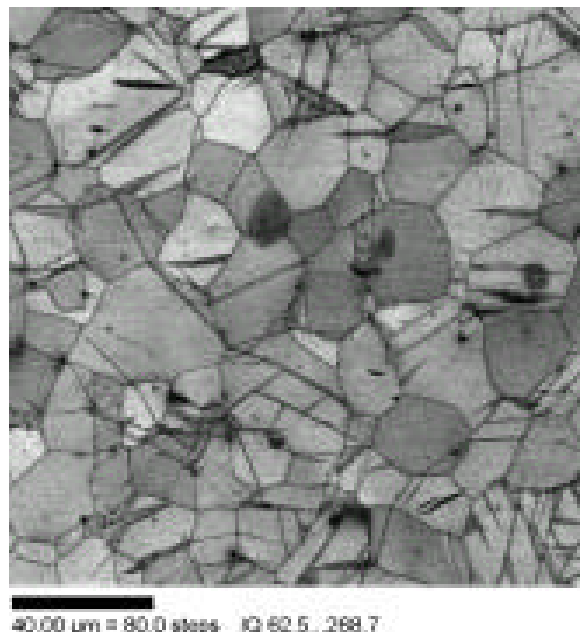

$52.00 \mathrm{~mm}=80.0$ skess $1062.5 .269 . ?$

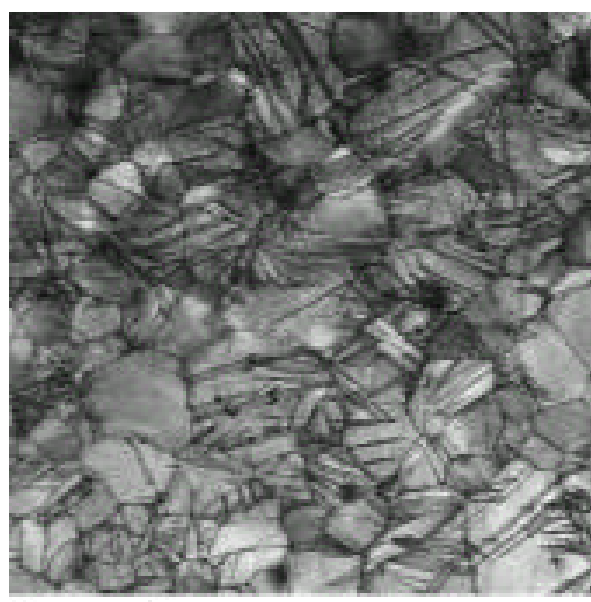

4900 j $17=70.0$ steps 1025.1 .299 .1

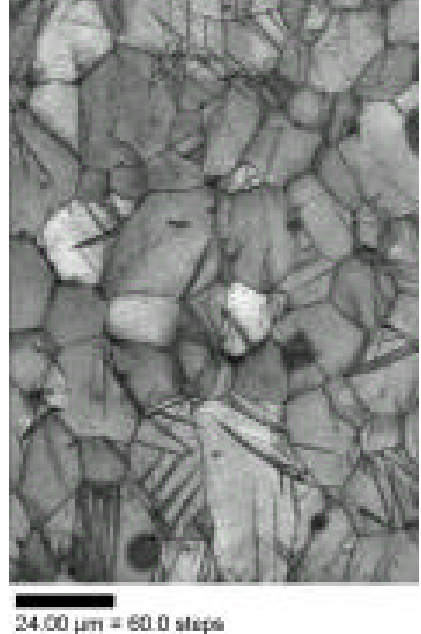

$24.00 \mathrm{um}=60.0$ stips

Figure 2 - Image quality maps from three zirconium samples with true strains of $4 \%$ in compression, $10 \%$ in compression and $16 \%$ in tension.

\begin{tabular}{lccc}
\hline Twin Type & $\begin{array}{c}\text { Sample 1 } \\
\text { 4\% compressive } \\
\text { strain }\end{array}$ & $\begin{array}{c}\text { Sample 2 } \\
\text { 10\% compressive } \\
\text { strain }\end{array}$ & $\begin{array}{c}\text { Sample 3 } \\
\mathbf{1 6 \%} \text { tensile } \\
\text { strain }\end{array}$ \\
\hline A $\{10 \overline{1} 2\}$ & 0.226 & 0.305 & 0.109 \\
B $\{11 \overline{2} 1\}$ & 0.100 & 0.073 & 0.053 \\
C $\{11 \overline{2} 2\}$ & 0.018 & 0.018 & 0.036 \\
\hline
\end{tabular}

Table 1 - Fraction of different twin boundary types in zirconium samples relative to all boundaries (greater than $2^{\circ}$ misorientation) in the sample. A tolerance of $10^{\circ}$ from the ideal misorientation was applied in these results.

Fig. 3 shows the distribution of $\Delta \omega$ vs. $\Delta \theta$ for the combined twin types in each sample as a function of strain. These distributions were calculated by binning the deviation space into $1^{\circ}$ bins in both $\Delta \omega$ and $\Delta \theta$. For each boundary in the scan $\Delta \omega$ and $\Delta \theta$ were calculated for each twin type and the appropriate bins incremented. Because the OIM data is collected on a regular grid, the longer boundaries are more accurately reconstructed from the grid than the shorter boundaries. In order to mitigate the effects of the shorter boundaries, the bins are incremented by the reconstructed boundary length. Each bin is then normalized to the total length of boundaries used in the binning process [10]. The effect of strain is evident in the compression samples by a shift toward greater $\Delta \theta$ and a broader distribution of $\Delta \omega$. This is acknowledged by the greater difficulty in discerning twin boundaries as the original twin grows and becomes incoherent, along with the effect of strain in deforming originally linear boundaries. These results, however, encourage the possibility of developing meaningful threshold values to discriminate between actual twin boundaries, including those that have since undergone deformation, and those that accidentally fulfill the $\Delta \theta$ requirements. Thus, the challenge is not to eliminate strained (and therefore deviated) boundaries from consideration if a characteristic can be identified. 

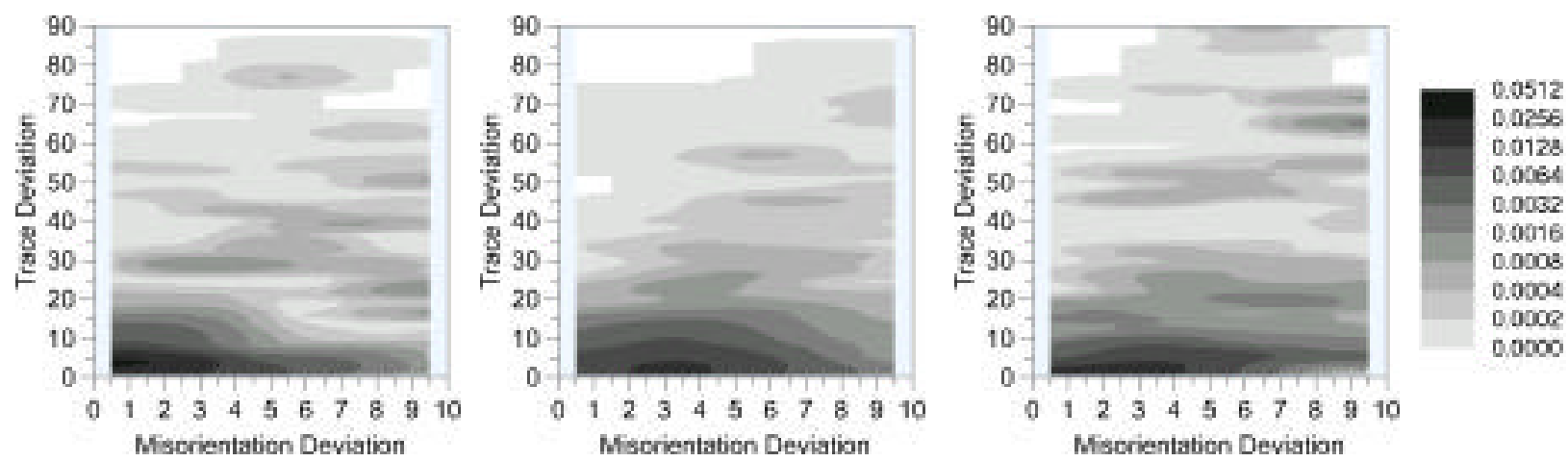

Figure 3 - Deviation distribution plots for all three twin types in zirconium samples.

Fig. 4 shows the same distribution plots for the twin types A, B, and C individually for the $10 \%$ compressed sample. The difference in distributions may be related to the characteristics of each of the twins. Twin type $\mathrm{A}$ is associated with a small twinning shear (0.17), relatively low activation stress, and typically exhibits large and sometimes irregularly shaped boundaries (i.e. significant deviations from coherency) [16]. This results in relatively loose constraints on acceptable boundary deviation. Conversely, the large twinning shear (0.63), higher activation stress, and narrow twin morphologies (i.e. small deviations from coherency) characterizing the other tension twin (twin B) may be expected to result in tighter constraints. These trends are reflected in the deviation distributions shown in Fig. 4 with respect to trace deviation, and suggest the possibility of tailoring the acceptable trace deviation threshold according to twin type. The misorientation deviation is apparently more sensitive to accumulated deformation than twin type. It is also noted that the compression twin (twin $\mathrm{C}$ ), which displays characteristics intermediate to $\mathrm{A}$ and $\mathrm{B}$, shows intermediate deviation behavior also.
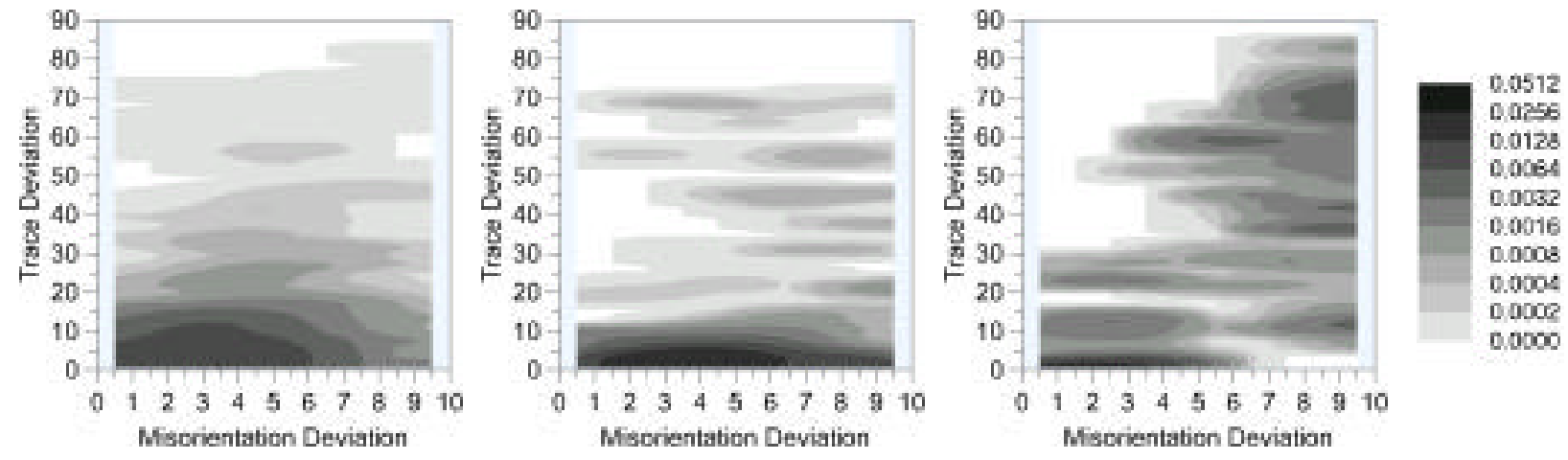

Figure 4 - Distribution plots for A, B and C twin types for the $10 \%$ compressed sample.

In order to characterize the twin deformation accurately, it is important to quantify the amount of twinning in the samples. The volume of twins can be determined by coupling the standard grain grouping algorithm employed in OIM [10]. However, when a neighboring pair of points is separated by a twin boundary they are considered to belong to the same grain. The constituent points of each OIM grain may now have distinctly different orientations. Once the grain grouping process has completed then each grain is analyzed for twin content. The dominant orientation in the grain is assumed to be the parent and the other orientations are assumed to belong to twin related 
daughters. It should be noted that, in deformed materials, such an approach can be an oversimplification as the dominant orientation does not necessarily correspond to the parent orientation. Twin fraction results from the deformed zirconium samples are given in Table 2.

\begin{tabular}{lcc}
\hline Sample & $\begin{array}{c}\text { Majority Rule } \\
\text { Misorientation }\end{array}$ & $\begin{array}{c}\text { Majority Rule } \\
\text { Misorientation + } \\
\text { Trace Analysis }\end{array}$ \\
\hline A 4\% Compression & 0.255 & 0.058 \\
B 10\% Compression & 0.506 & 0.104 \\
C 16\% Tension & 0.331 & 0.171 \\
\hline
\end{tabular}

Table 2 - Twin fractions in zirconium calculated with and without the trace deviation analysis. A tolerance of $10^{\circ}$ was allowed for both deviation parameters.

\section{Recrystallization Twins}

Previous applications of the trace deviation analysis $[9,10]$ on fcc recrystallization twins showed deviation distributions where the cluster of twin boundaries extended from 0 to about $10^{\circ}$ along the misorientation deviation axis and clustered at $0^{\circ}$ on the trace deviation axis but had significant scatter up to almost $90^{\circ}$. There was some evidence in these results that suggested that the scatter at larger trace deviations was due to error in the boundary trace measurements in the OIM scans. In order to confirm the effect of the boundary trace reconstruction OIM data from OIM scans on the same material at different step sizes were performed. Similar calculations have been made on data from a recrystallized nickel sample and are shown in Fig. 5.
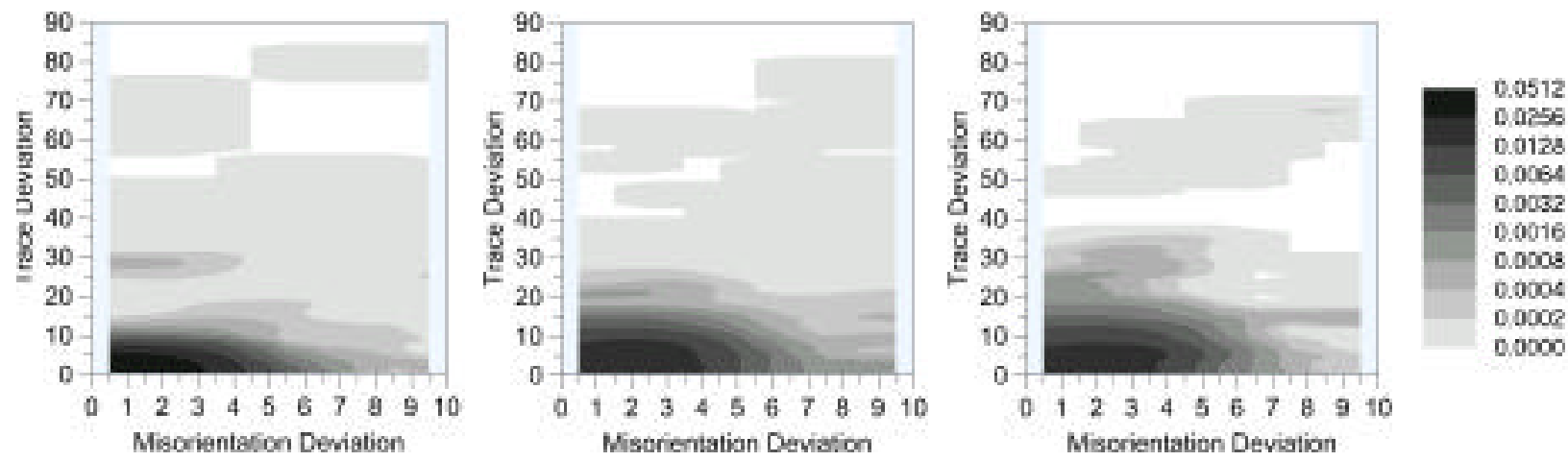

Figure 5 - OIM scans from a recrystallized nickel sample with scan step sizes of 1, 5 and $10 \mu \mathrm{m}$ (x, y and $\mathrm{z}$ measurement points respectively).

It is clear from these plots that, as the scan density increases, the spread in the cluster along the trace deviation direction decreases.

\section{Summary}

An advanced twin characterization analysis for EBSD-generated data was applied to zirconium and nickel to explore its potential for classification and property evaluation for both deformation and recrystallization twins. The judicious application of trace deviation distribution data for both twin 
types may provide a more discriminating means of distinguishing between actual and 'accidental' twins originally identified by misorientation criteria alone.

\section{References}

[1] B.L. Adams, S.I. Wright and K. Kunze: Met. Trans. Vol. 24A (1993), p. 819.

[2] T. Watanabe: Grain Boundary Architecture for High Performance Materials (Eds. R. C. Pond, W. A. T. Clark and A. H. King, The Minerals, Metals and Materials Society, Warrendale, Pennsylvania 1998), p. 19.

[3] V. Randle: Acta Met. Mat. Vol. 42 (1994), p. 1769.

[4] E.M. Lehockey, G. Palumbo and P. Lin: Effect of Grain Boundary Structure in Reducing Susceptiblity of Lead-Acid Battery Grids to Corrosion, Creep and Cracking (Eds. R. C. Pond, W. A. T. Clark and A. H. King, The Minerals, Metals and Materials Society, Warrendale, Pennsylvania 1998), p. 45.

[5] Y. Pan, B.L. Adams, T. Olson and N. Panayotou: Acta Mat. Vol. 44 (1996), p. 4685.

[6] V. Randle, M. Caul and J. Fiedler: Microscopy and Microanalysis Vol. 3 (1997), p. 224.

[7] V. Randle and D.J. Dingley: Scripta Metallurgica Vol. 23 (1989), p. 1565.

[8] D.P. Field and B.L. Adams: Met. Trans. Vol. 23 (1991), p. 2515.

[9] S.I. Wright: Journal of Electronic Materials Vol. 31 (2002), p. 50.

[10]S.I. Wright and R.J. Larsen: Journal of Microscopy (2002), in press.

[11]V. Randle: Scripta Materialia Vol. 44 (2001), p. 2789.

[12]D.G. Brandon: Acta Metallurgica Vol. 14 (1966), p. 1479.

[13]J.F. Bingert, T.A. Mason, G.C. Kaschner, P. Maudlin and G.T. Gray, III: Met. Mat. Trans. (2002), in press.

[14]G.C. Kaschner, J.F. Bingert, C. Liu, M.L. Lovato, P.J. Maudlin, M.G. Stout and C. Tome: Acta Mat. Vol. 49 (2001), p. 3097.

[15]T.A. Mason, J.F. Bingert, G.C. Kaschner, S.I. Wright and R.J. Larsen: Met. Mat. Trans. (2002), in press.

[16]R.E. Reed-Hill: (Eds. R. E. Reed-Hill, J. P. Hirth and H. C. Rogers, TMS, Warrendale, PA 1964), p. 295. 